\title{
GEORGIA ELECTRICITY BALANCE DURING THE YEARS OF STATE INDEPENDENCE (1989-2019)
}

\author{
DAVID NARMANIA \\ Doctor of Economics, Professor \\ Ivane Javakhishvili Tbilisi State University, Georgia \\ d.narmania@gnerc.org
}

\section{DEMUR CHOMAKHIDZE}

Doctor of Economic Sciences, Professor

Georgian Technical University, Georgia

demurchomakhidze@yahoo.com

Abstract. The article analyzes the electricity balance of Georgia during the 30 years of independence (1989-2019). It is said to be in deficit throughout this period except 2010. The deficit was caused by the fact that during the analysis period all the components of the balance deteriorated, primarily electricity production. The latter was at a record low in 2005, when the annual deficit exceeded 1.2 billion kWh. The excess of electricity in the balance was conditioned by the record output in the HPPs as well. It is noteworthy that in recent years, electricity consumption is growing much faster than production. Due to this, in 2019 the maximum amount of deficit was almost 1.4 billion kWh. The paper sets out ideas for improving the situation.

KEYWORDS: ELECTRICITY BALANCE, ELECTRICITY GENERATION, ELECTRICITY CONSUMPTION, EXPORT, IMPORT.

For citation: Narmania D., Chomakhidze D. (2020). Georgia electricity balance during the years of state independence (19892019). Globalization and Business, №9, pp. 197-202 (In Georgian). https://doi.org/10.35945/gb.2020.09.024 


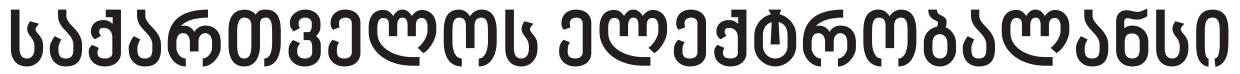

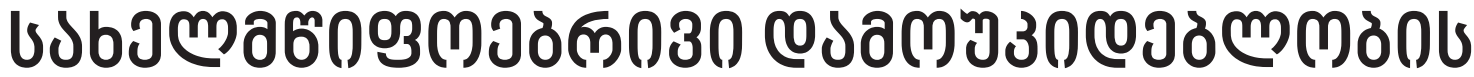 бщコวว0 (1989-2019)}

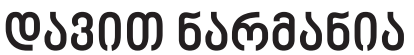

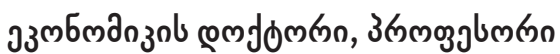

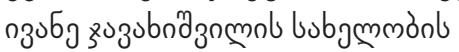

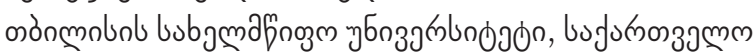

d.narmania@gnerc.org

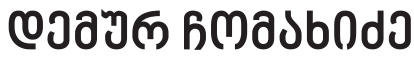

эзмбмапзи

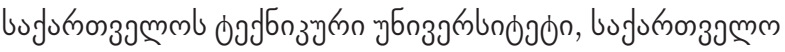

demurchomakhidze@yahoo.com

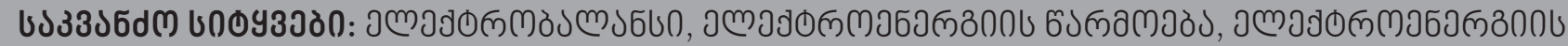

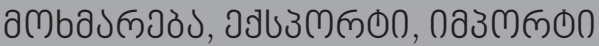

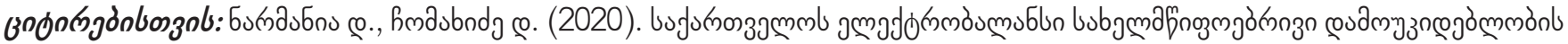

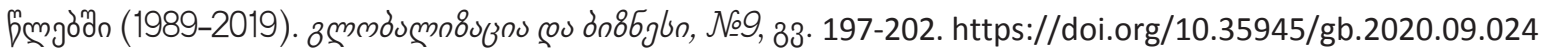

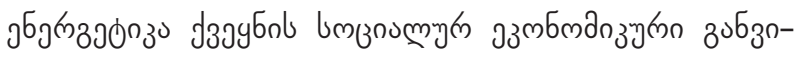

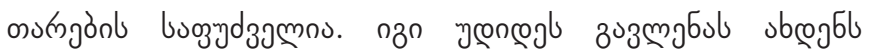

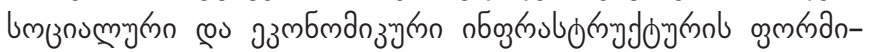

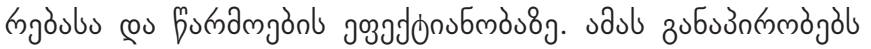

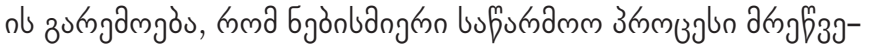

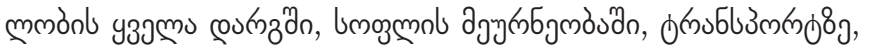

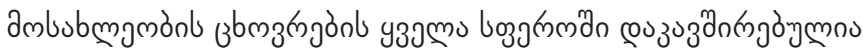

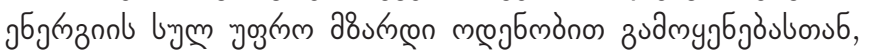

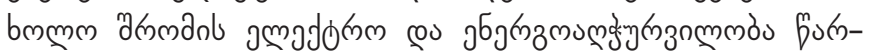
amu@

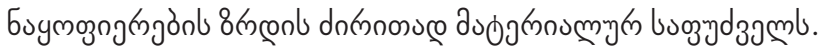

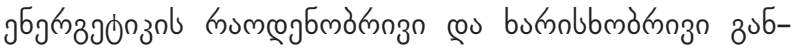

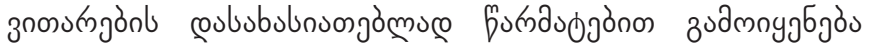

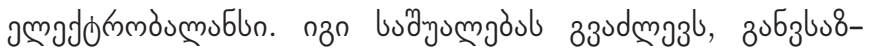

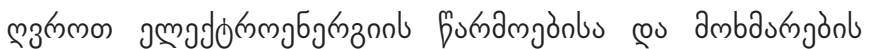

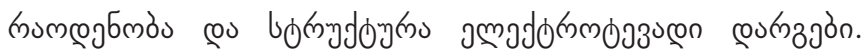

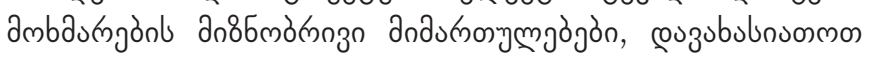

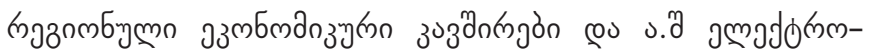

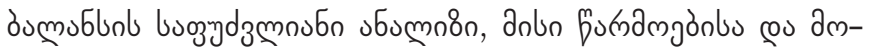

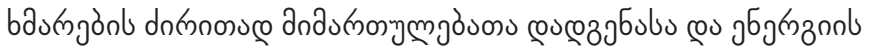

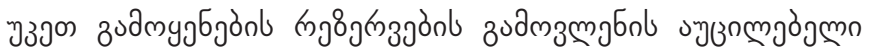

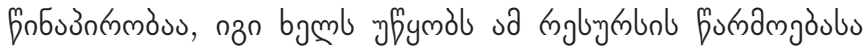

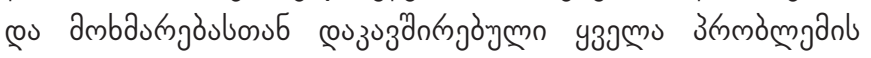

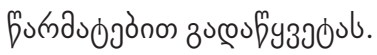

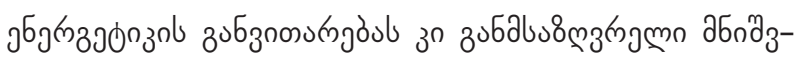

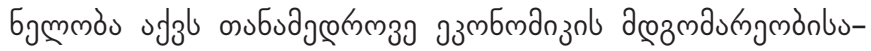

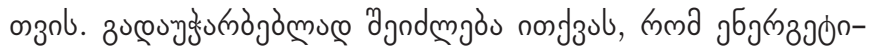

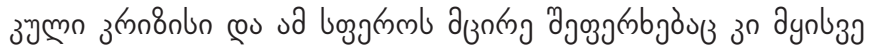

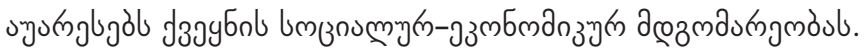

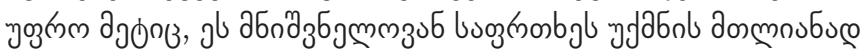

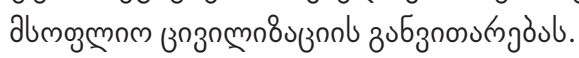

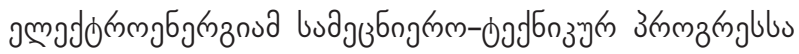

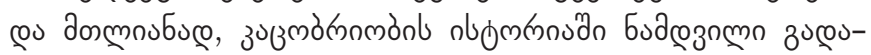

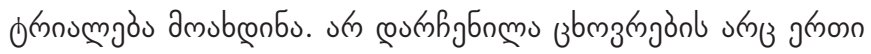

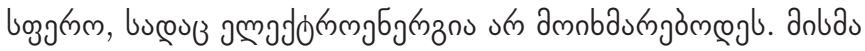

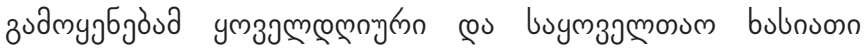

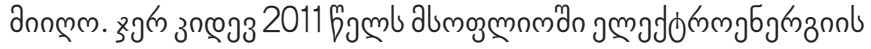

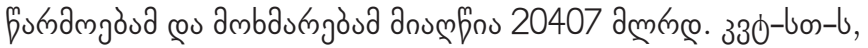

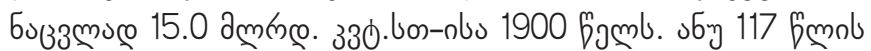

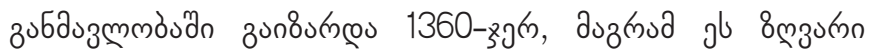

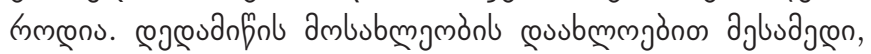

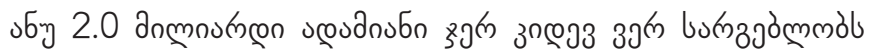

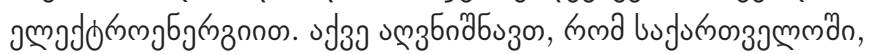

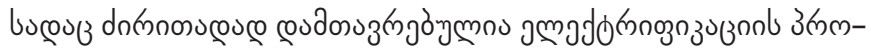

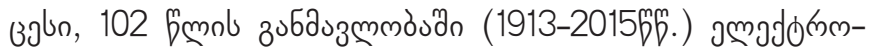
jб

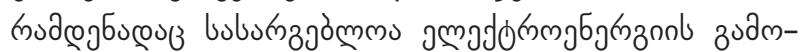

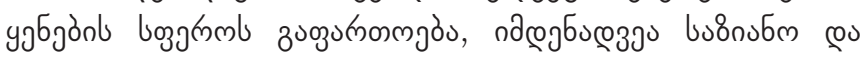




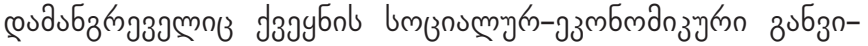

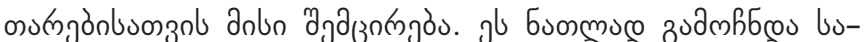

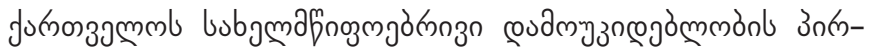

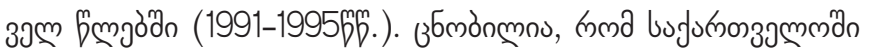

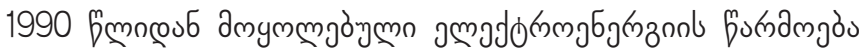

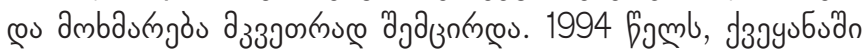

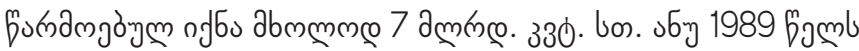

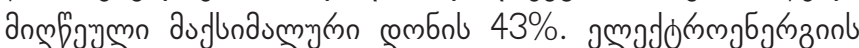

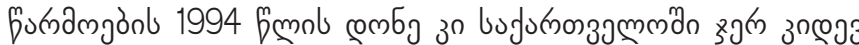

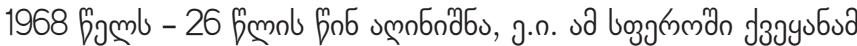

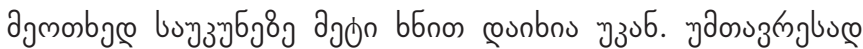

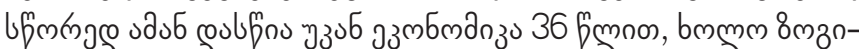

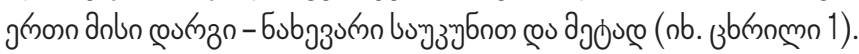

ju ojufon ndm

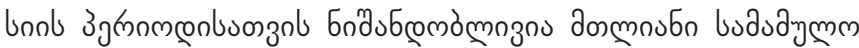

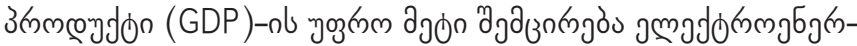

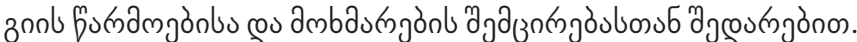

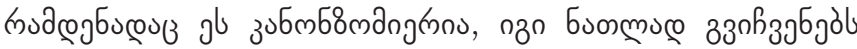

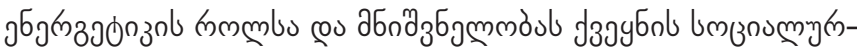

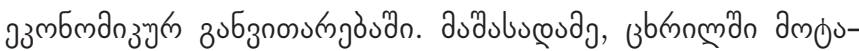

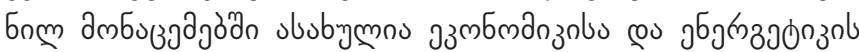

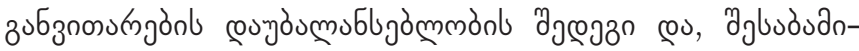

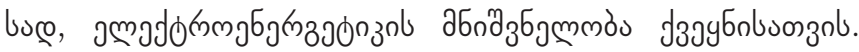

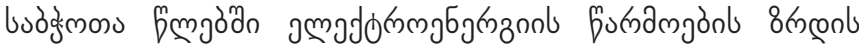

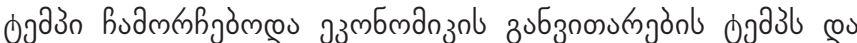

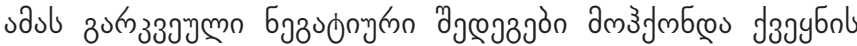

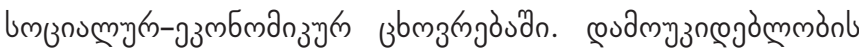

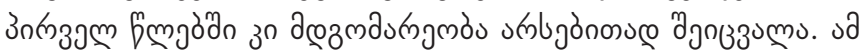

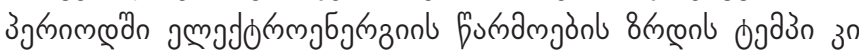

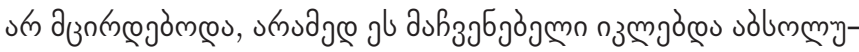

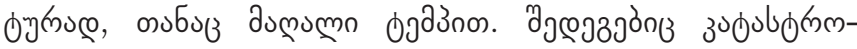

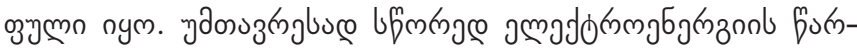

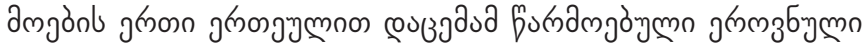

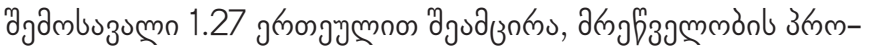

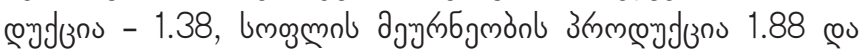

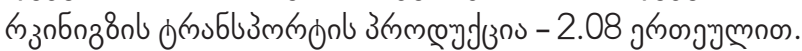

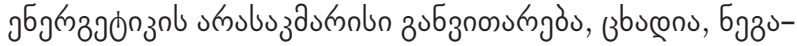

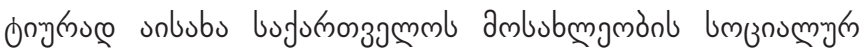

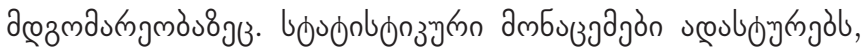

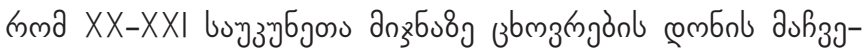

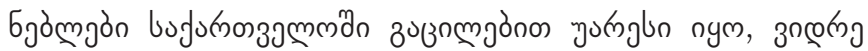

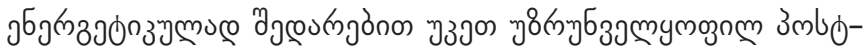

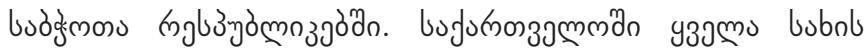

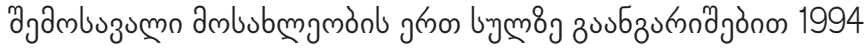

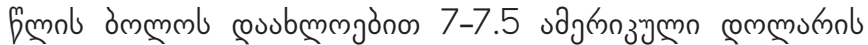

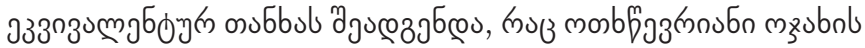

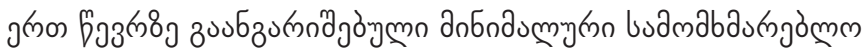

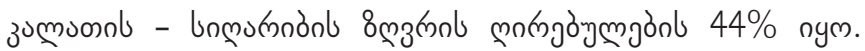

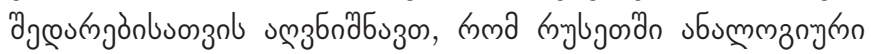

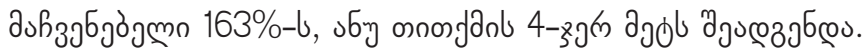

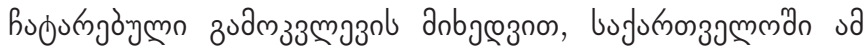

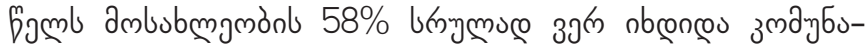

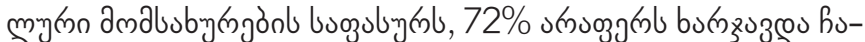

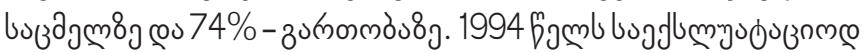

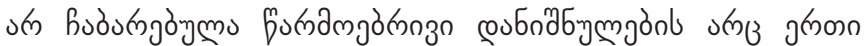

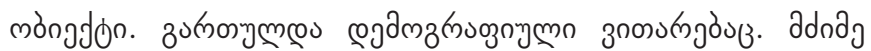

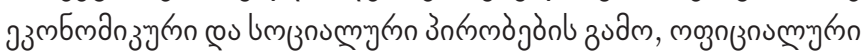

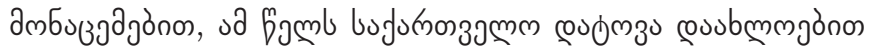

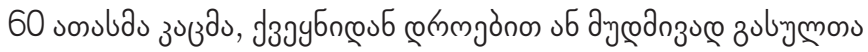

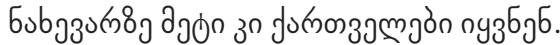

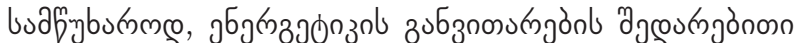

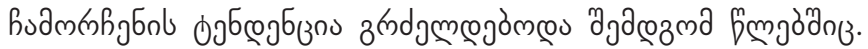

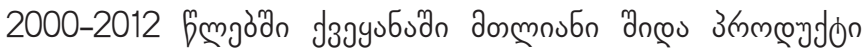

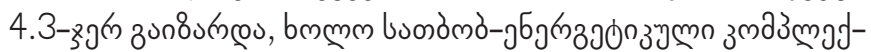

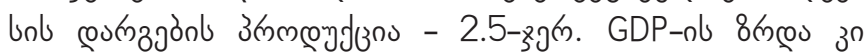

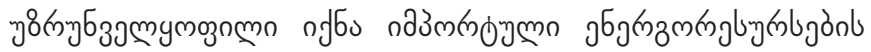

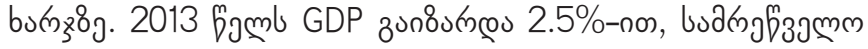

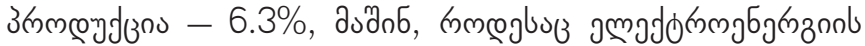

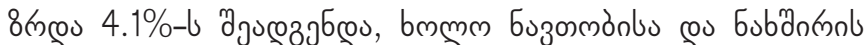

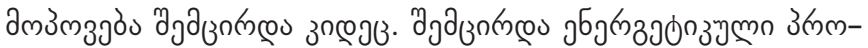

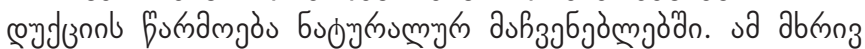

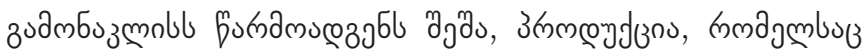

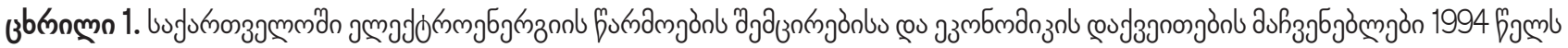

\begin{tabular}{|c|c|c|c|}
\hline 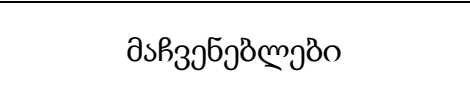 & 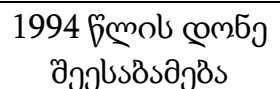 & 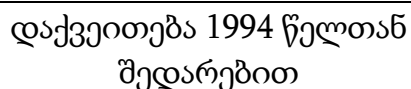 & 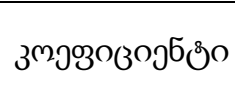 \\
\hline 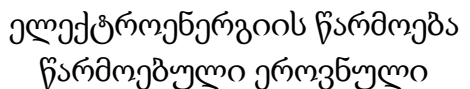 & 1968 fombl & 26 ดวмก & 1.0 \\
\hline gुambsзs sмо & 1961 6jomb & 33 бэмоо & 1.27 \\
\hline 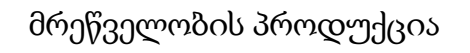 & 1958 bomls & 36 бэмео & 1.38 \\
\hline 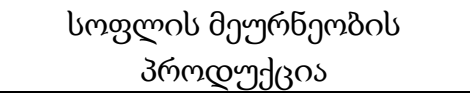 & 1945 bombl & 49 ஜூตฺо & 2.08 \\
\hline 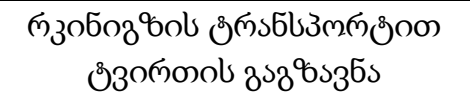 & 1940 60mb & 54 бэмо & 2.08 \\
\hline 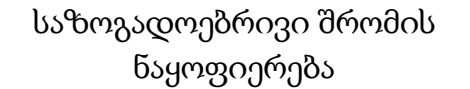 & 1961 bomls & 33 бэмо & 1.27 \\
\hline
\end{tabular}




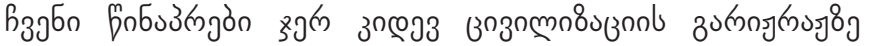

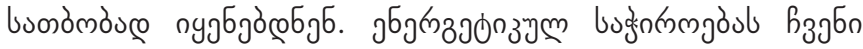

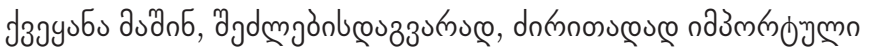

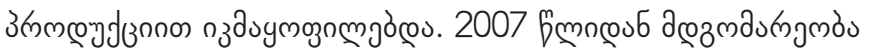

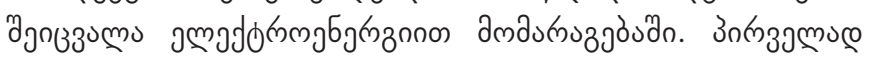

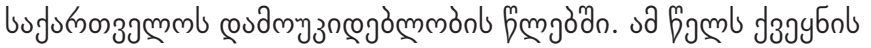

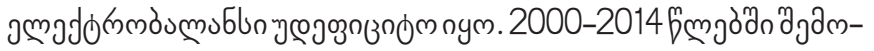

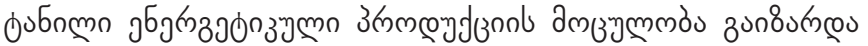

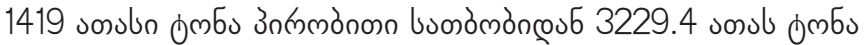

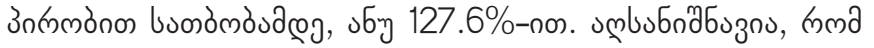

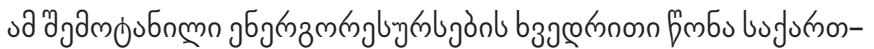

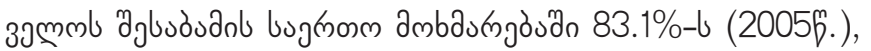

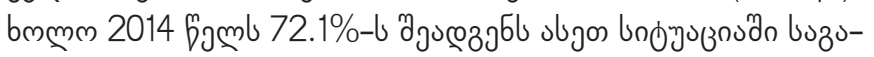

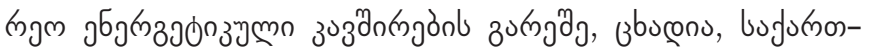

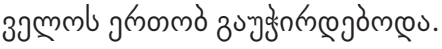

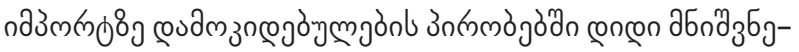

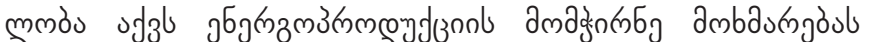

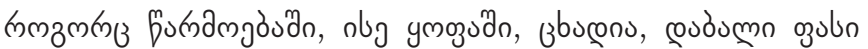
3j

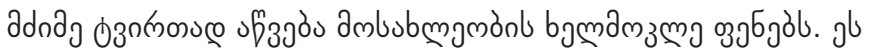

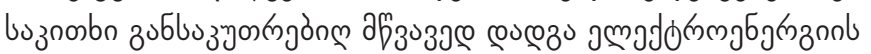

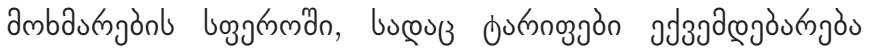

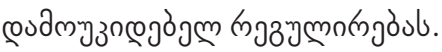

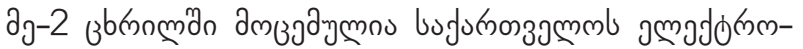

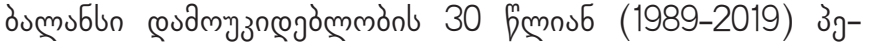

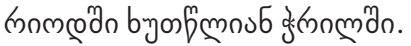

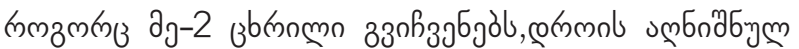

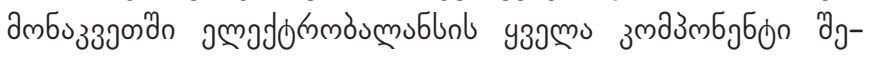

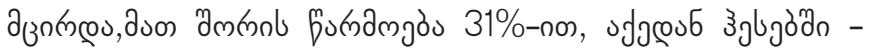

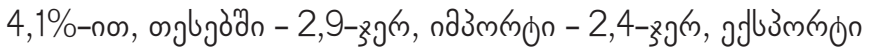

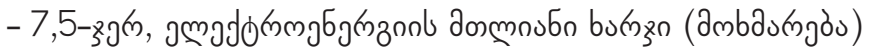

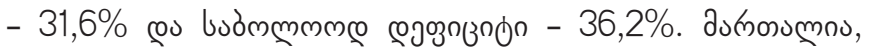

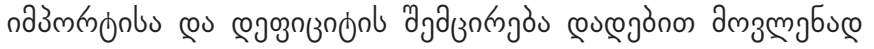

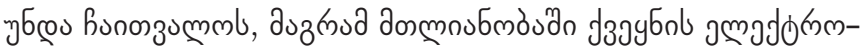

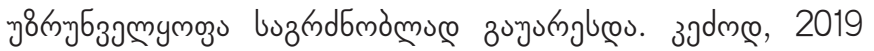

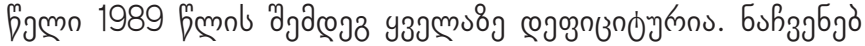

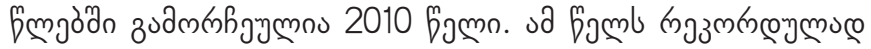

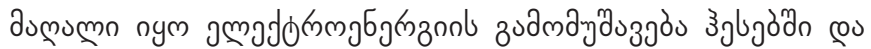

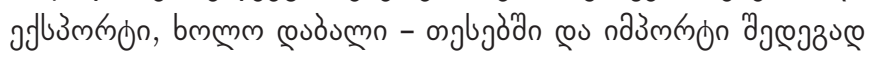

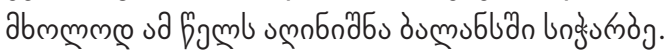

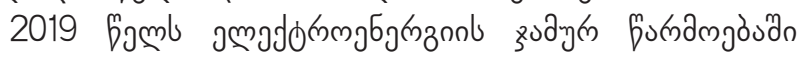

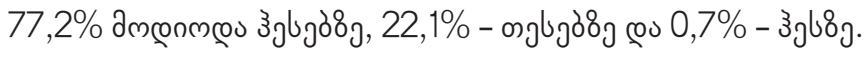
sa fambl bughnmm 8ubeo 1626,5 amb 330. bon-n J3aybn

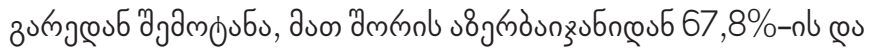

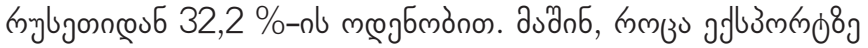

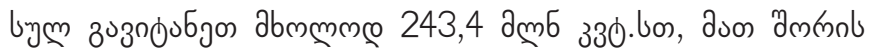

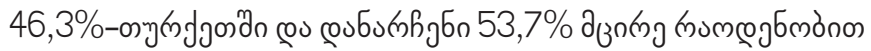

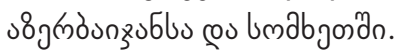

абпдзб jmadd

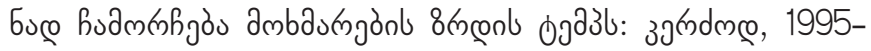

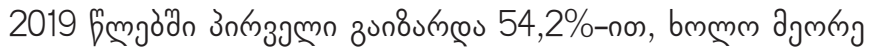

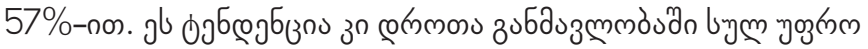

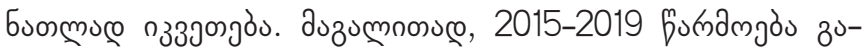

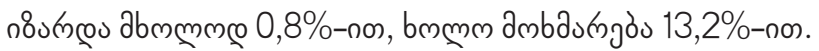

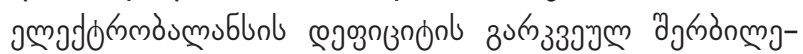

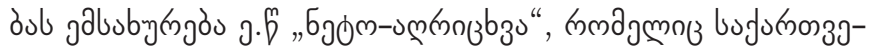

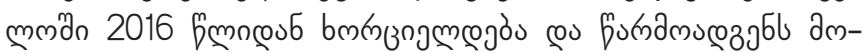

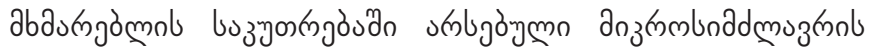

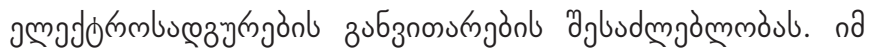

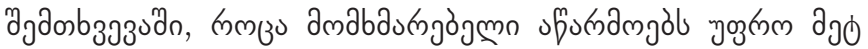

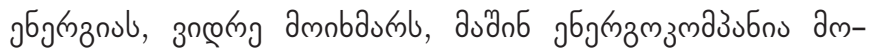

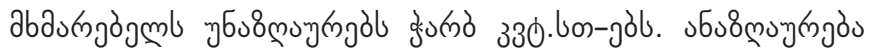

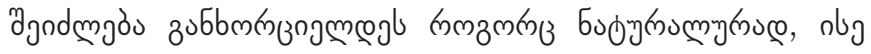

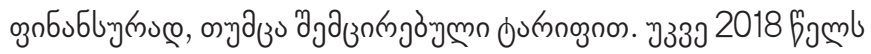

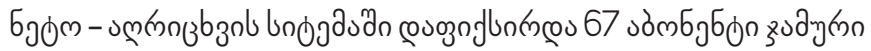
unadmuz zinnon 739,7 330.

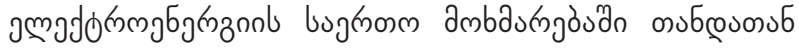

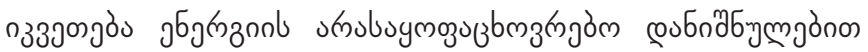

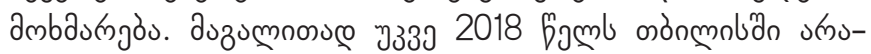

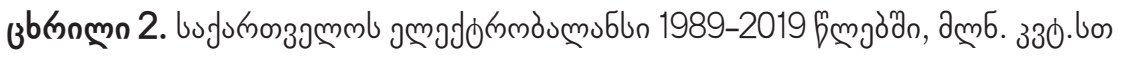

\begin{tabular}{|c|c|c|c|c|c|c|c|c|}
\hline 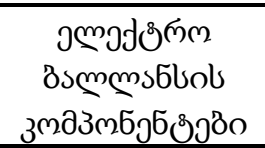 & 1989 & 1995 & 2000 & 2005 & 2010 & 2015 & 2019 & $\begin{array}{c}\text { 20196, \%-oo } \\
\text { 19896,-ons6 }\end{array}$ \\
\hline 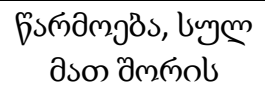 & 15825,0 & 7082,0 & 7451,3 & 6880,8 & 9919,2 & 10832,5 & 10925,9 & 69,0 \\
\hline Зృlo & 8787,0 & 6383,0 & 5952,1 & 6041,9 & 9263,3 & 8453,8 & 8428,4 & 95,9 \\
\hline ojlo & 7038,0 & 699,0 & 1499,2 & 838,9 & 655,9 & 2378,7 & 2420,2 & 34,3 \\
\hline dgloo & - & - & - & - & - & - & 77,3 & - \\
\hline одзмпљо & 3999,5 & 754,1 & 599,5 & 1399,0 & 222,1 & 699,3 & 1626,5 & 40,6 \\
\hline 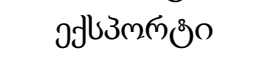 & 1839,2 & - & 204,6 & 120,0 & 1524,3 & 659,3 & 243,4 & 13,2 \\
\hline 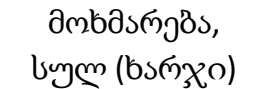 & 17985,3 & 7836,1 & 7846,2 & 8159,8 & 8617,0 & 10871,9 & 12309,0 & 68,4 \\
\hline Bsmsblso (+;-) & $-2160,3$ & $-754,1$ & $-394,9$ & $-1279,0$ & 1302,2 & $-39,4$ & $-1383,1$ & 63,9 \\
\hline
\end{tabular}




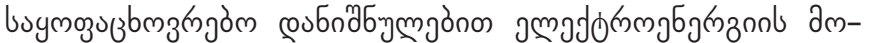

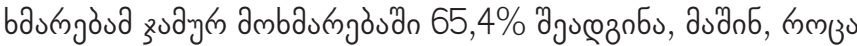

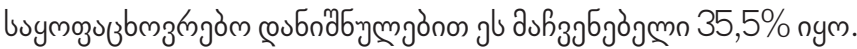

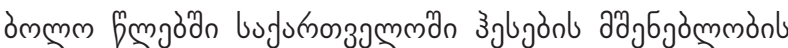

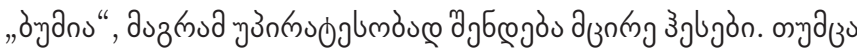

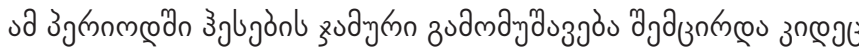

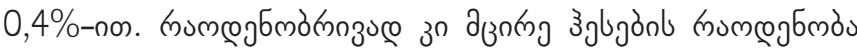

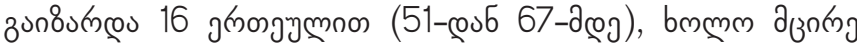

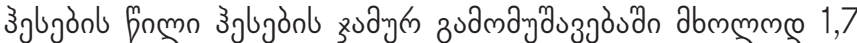

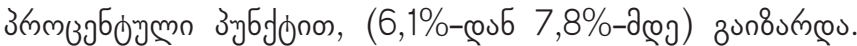

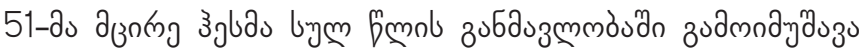

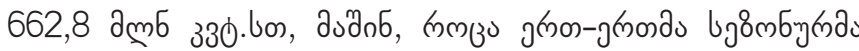
зु

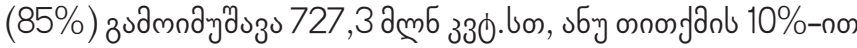

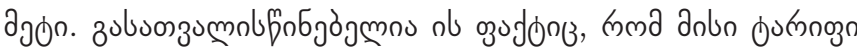

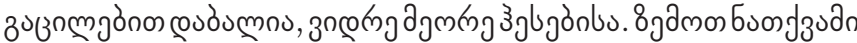

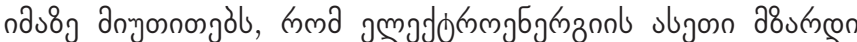

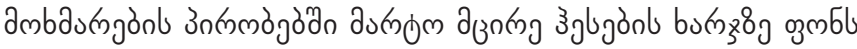

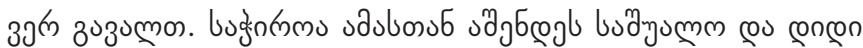

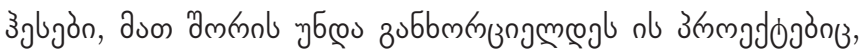

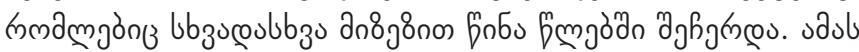

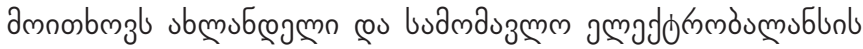
ง ๓l

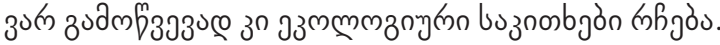

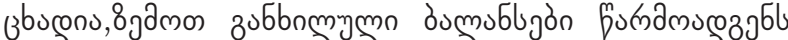

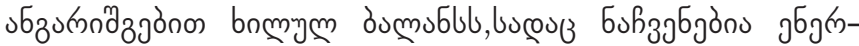

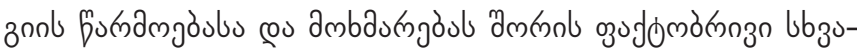

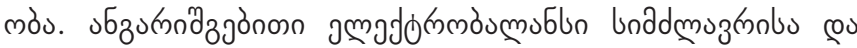

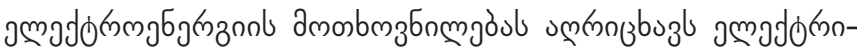

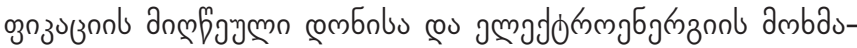

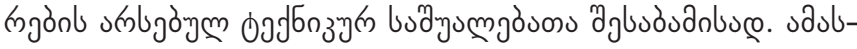

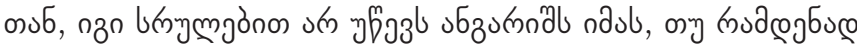

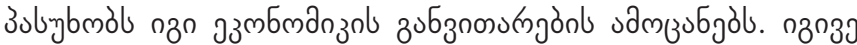

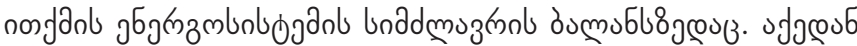

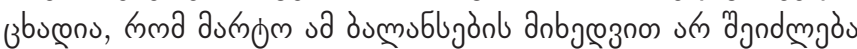

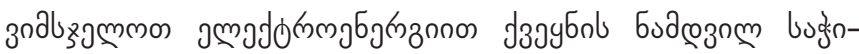

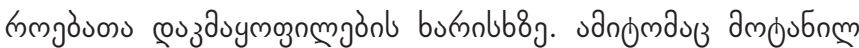

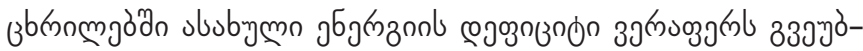

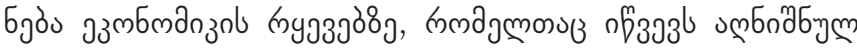

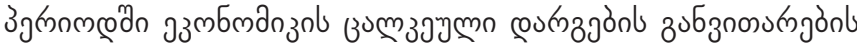

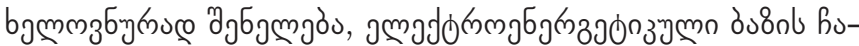
ammhjбol zudm.

jбgr

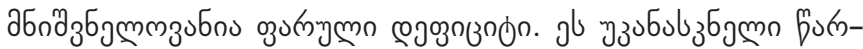

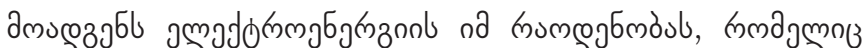

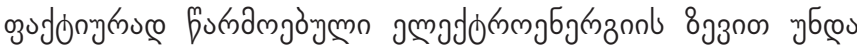

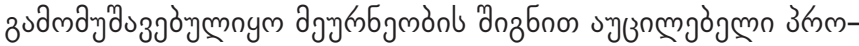

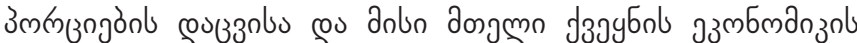

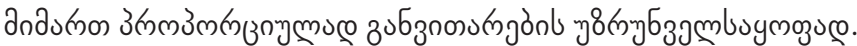

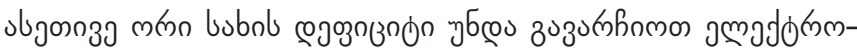

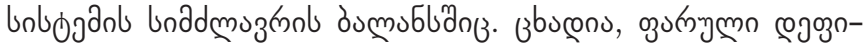

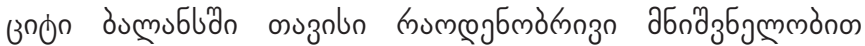

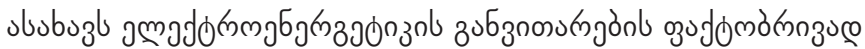

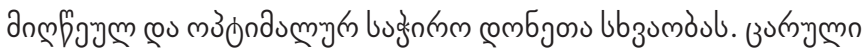

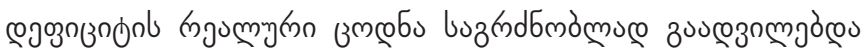

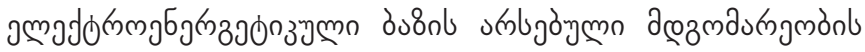

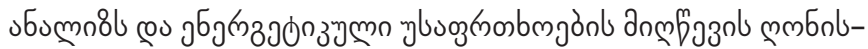

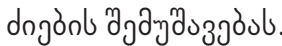

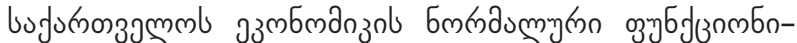

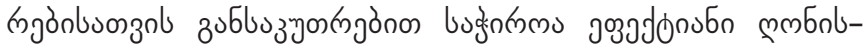

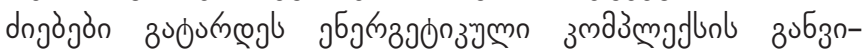

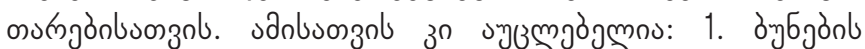

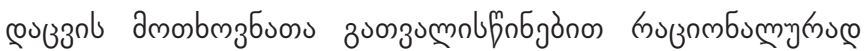

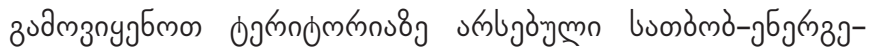

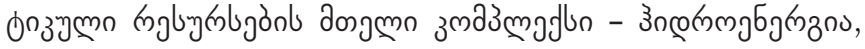

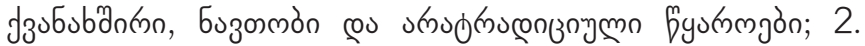

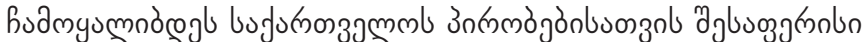
buondmo-gб

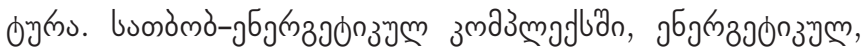

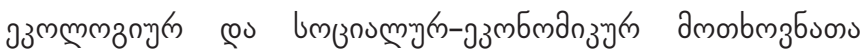

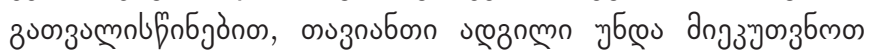

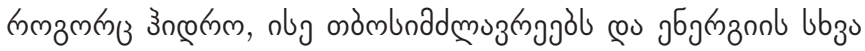

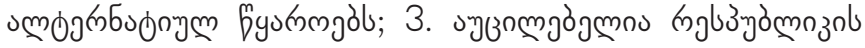

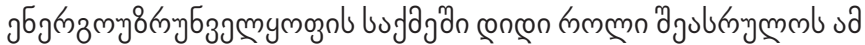

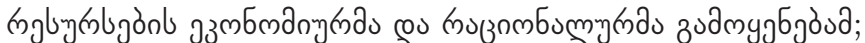

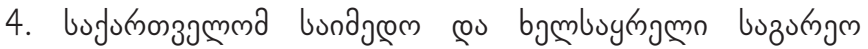

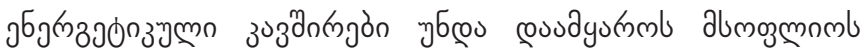

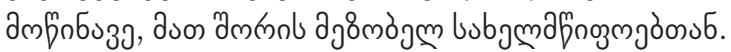

coumznl a a

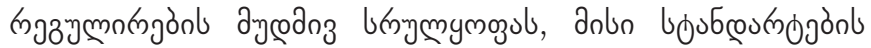

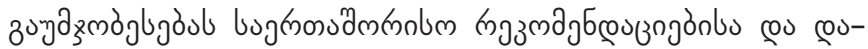

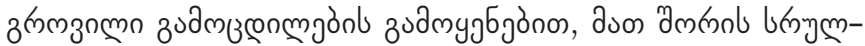

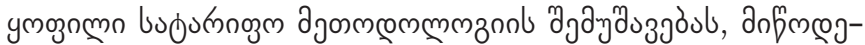

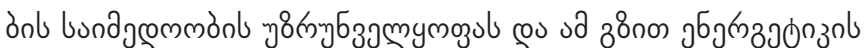
ugdom

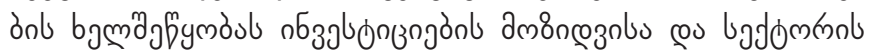

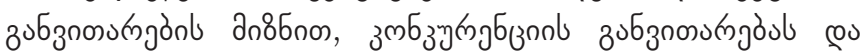

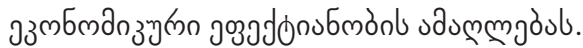

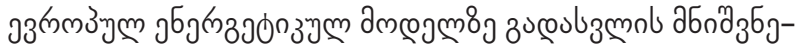

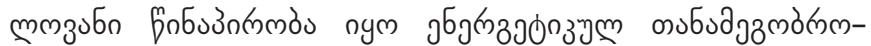

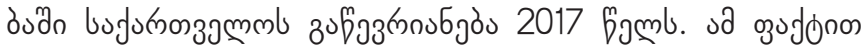

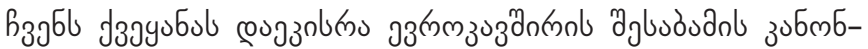

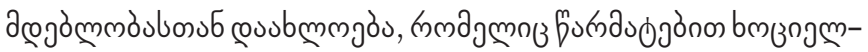

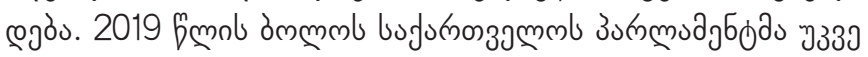

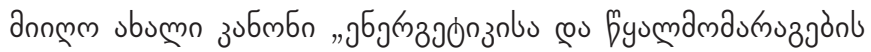

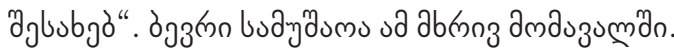

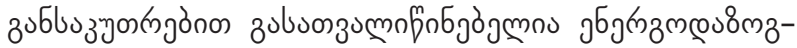

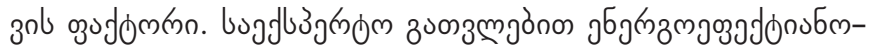

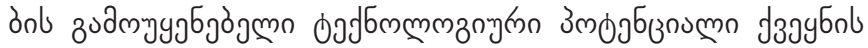

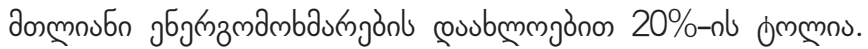




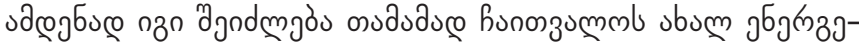
onzym malughloce.

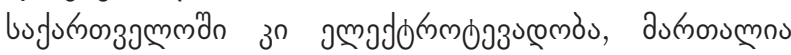

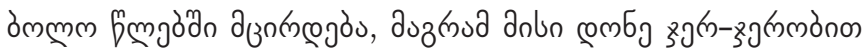

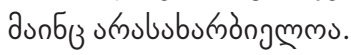

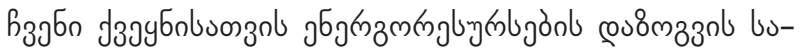

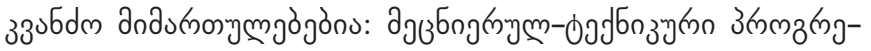

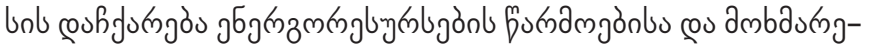

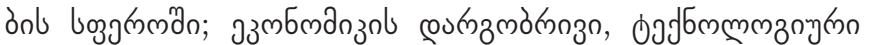

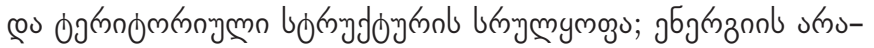

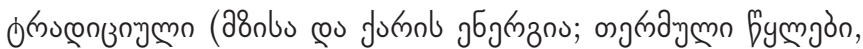

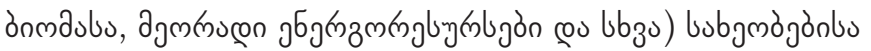

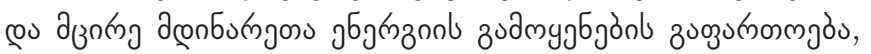

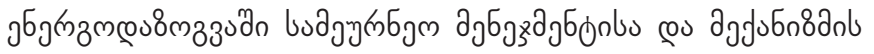
uñymymozo.

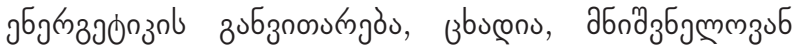

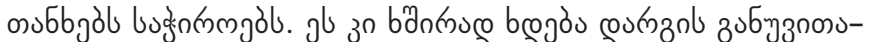

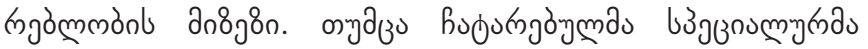

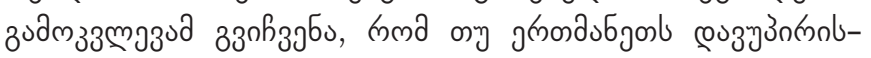

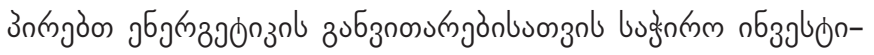

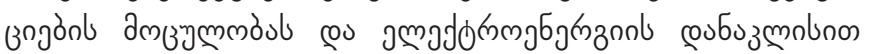

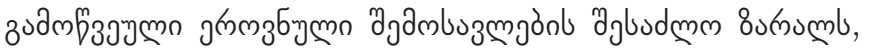

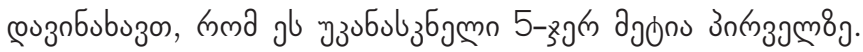

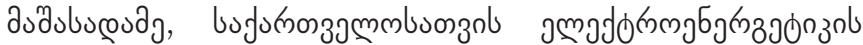

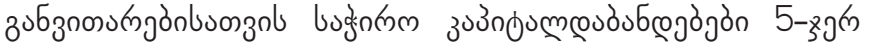

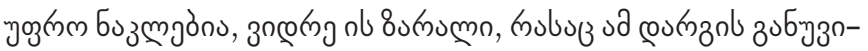

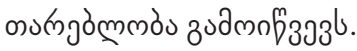

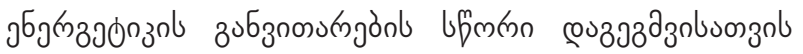

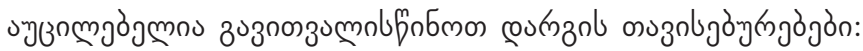

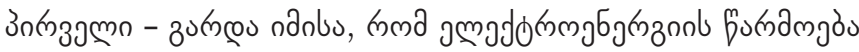

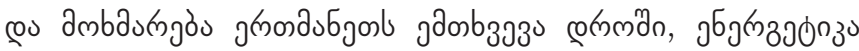

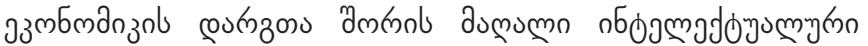

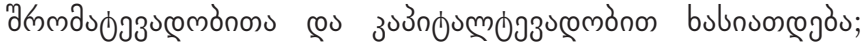

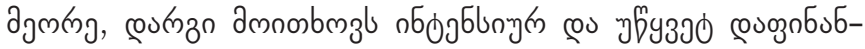

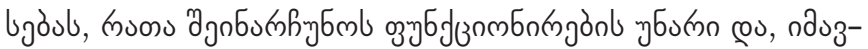

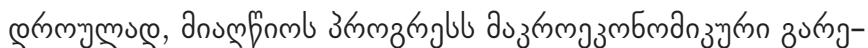

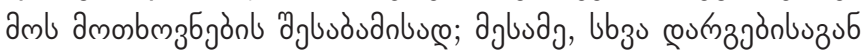

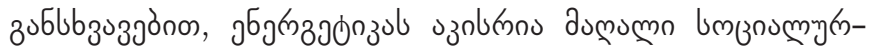

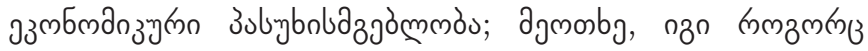

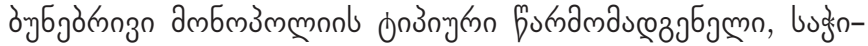

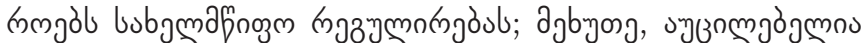

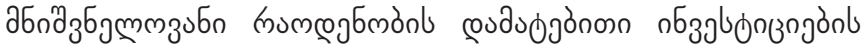

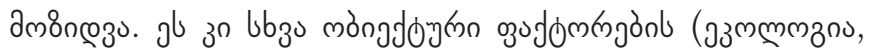

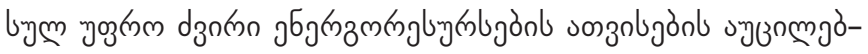

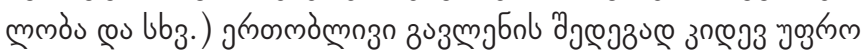

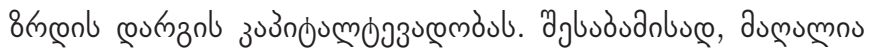

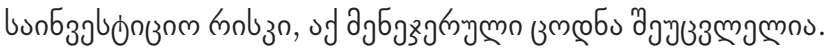

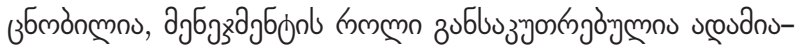

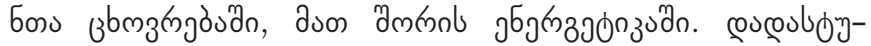

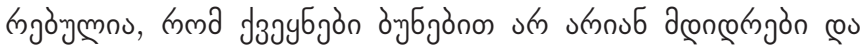

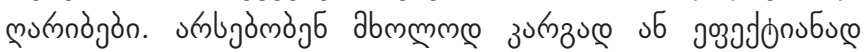

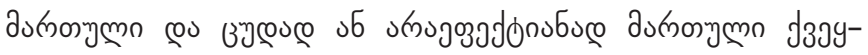

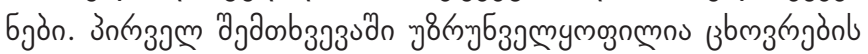

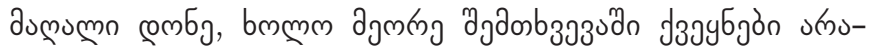

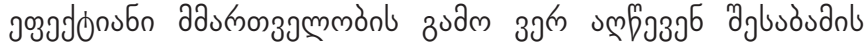

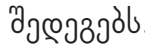

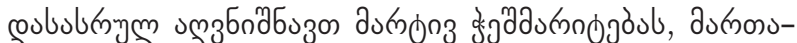

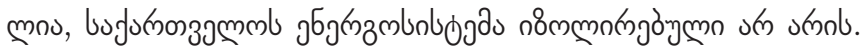

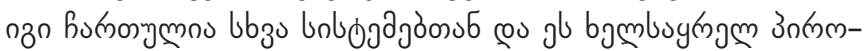

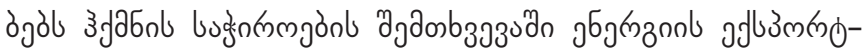

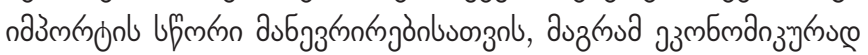

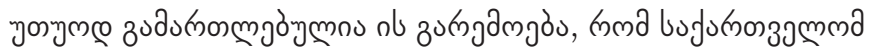

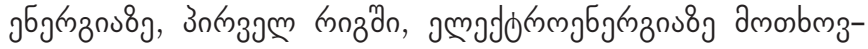

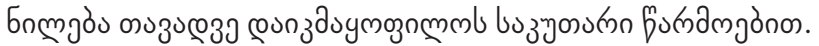

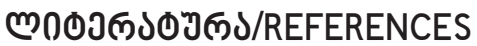

Chomakhidze, D. (2007). Energetic Balance of Georgia. Tbilisi. (In Georgian)

Chomakhidze, D., Tskhakaia, Q. (2019). The "Tendencies" of formation of main parameters of Georgia electro-energetic balance. Globalization and Business, №7.

Chomakhidze, D., Narmania, D. (2018). Ecological Challenges in the Development of Georgian Energetics. Globalization and Business, №5.

Chomakhidze, D. Narmania, D. (2018). Synthetic Menegment of Energy and Ecology in Georgia, AASCIT (American Association for Science and Technology.

Geostat - Energy Balance of Georgia, 2013-2019.

GNERC Annual Reports, 1998-2018.

ESCO Annual Reports, www.esco.ge 